\title{
IDENTIFYING CHROMOSOME REARRANGEMENTS IN THE ALLOPOLYPLOID BRASSICA NAPUS USING PYROSEQUENCING
}

\author{
A Thesis \\ presented to \\ the Faculty of California Polytechnic State University, \\ San Luis Obispo
}

\author{
In Partial Fulfillment \\ of the Requirements for the Degree \\ Master of Science in Biology
}

by

Alexandra Rose Barbella

November 2013 
(C) 2013

\section{Alexandra Rose Barbella}

ALL RIGHTS RESERVED 


\section{COMMITTEE MEMBERSHIP}

TITLE: Identifying Chromosome Rearrangements In The Allopolyploid Brassica Napus Using Pyrosequencing
AUTHOR:
Alexandra Rose Barbella

DATE SUBMITTED: November 2013

COMMITTEE CHAIR: $\quad$ Ed Himelblau, PhD Associate Professor of Biology

COMMITTEE MEMBER: Ken Hillers, PhD Associate Professor of Biology

COMMITTEE MEMBER: Charles Knight, PhD Associate Professor of Biology 


\author{
ABSTRACT \\ Identifying Chromosome Rearrangements In The \\ Allopolyploid Brassica Napus Using \\ Pyrosequencing
}

\begin{abstract}
Allopolyploids form through the hybridization of two or more diploid genomes. A challenge to reproduction in allopolyploids is that pairing can occur between homologous chromosomes or homeologous chromosomes (i.e.different subgenomes.). Crossover between homeologous chromosomes can result in chromosome rearrangements that lower fertility and overall fitness.

Rearrangements can alter the dosage of either entire chromosomes or just parts of chromosomes. Understanding the frequency and extent of rearrangements will help to explain the evolution and genome stabilization of agriculturally important allopolyploid species. Pyrosequencing is a useful tool in the study dosage changes in allopolyploids because it allows quantification of the relative contribution from each progenitor species at any given locus. Here we use pyrosequencing to analyze resynthesized Brassica napus allopolyploids and their progeny. Targets for pyrosequencing were identified using a bioinformatic approach taking advantage of recently-released Brassica genome sequence. SNPs identified through bioinformatics were confirmed through molecular biology. Markers along the A3/C3 homeolog pair were used to identify the occurrence of novel homeologous exchanges during meiosis in the parent plant, and segregation patterns arising from dosage changes in the parent. We identify a higher frequency of homeologous rearrangements at the distal end of the chromosomes. We also observe that the presence of a dosage change in a parent increases the likelihood that the chromosome bearing the dosage change will undergo subsequent rearrangements in neighboring loci.
\end{abstract}

Key Words: polyploid, bioinformatics, pyrosequencing 


\section{ACKNOWLEDGMENTS}

This work was supported by The California Polytechnic State University

Extramural Funding Initiative. We are grateful to Patrick Edger at University of

Missouri, Columbia, for providing Brassica genome sequence; Jen Vanderkelen and Joshua Dillard for pyrosequencing training and advice. 


\section{TABLE OF CONTENTS}

List of Figures

viii

Chapter

I. INTRODUCTION

a. Species Formation 1

b. Brassica napus as a Model Organism___ 5

C. Approaches to Study Chromosome Rearrangements __ 6

d. Previous Research _ 12

$\begin{array}{ll}\text { II. METHODS } & 14\end{array}$

a. Greenhouse _ 14

b. DNA Extraction and Sequencing __ 14

c. Search for Non-Orthologous or -Paralogous genes __ 15

d. Amplification and Sequencing of Candidate Markers __ 16

e. Identification and Validation of SNPs__ 17

$\begin{array}{ll}\text { f. Pyrosequencing _ } & 17\end{array}$

g. Statistical Analysis of Pyrosequencing Data__ 19

III. RESULTS_ 20

a. Dosage Changes At Loci Along The A3:C3 Homeologs__ 20

b. Frequency Of Novel Rearrangements __ 23

c. Segregation Of Rearrangements Present In The Parent_ 23

IV. DISCUSSION_ 25

a. Allopolyploidy in Brassica napus__ 25 
b. Inheritance of Homeologous Exchanges

c. Frequency of Homeologous Exchange on an Acrocentric Chromosome 28

V. CONCLUSION 30 REFERENCES 31 APPENDIX 38 


\section{LIST OF FIGURES}

Figure 1 Allopolyploid Formation

Figure 2 Possible Gametes Produced Through Cross-Overs In Tetraploids During Meiosis I

Figure 3 Different Types Of Chromosomal Rearrangements That Can

Arise From Pairing Between Homeologous Chromosomes

Figure 4 "The Polyploid Ratchet"

8

Figure 5 SNPs 10

Figure 6 Example Of A Pyrosequence Across A SNP-Containing

Region 12

Figure 7 Chromosome Diagrams Of Parents And Their

Progeny 22

Figure 8 Mendelian and Non-Mendelian Segregation For Parent \#3 29 


\section{CHAPTER I: INTRODUCTION}

\section{Species Formation}

When two different species mate they can produce hybrid offspring that are genetically and phenotypically distinct from themselves. Hybridization occurs in most eukaryotes, and it is common in plants. Hybridization between plant species followed by whole genome duplication (WGD) results in offspring with more than two complete sets of chromosomes. Since the newly created hybrid species contains chromosomes from two different progenitor species, it is referred to as an allopolyploid. Allopolyploid plants form naturally and many agricultural crops are known to be allopolyploid (Ozkan et al., 2001; Kim et al., 2008; Lim et al., 2008; Pontes et al., 2004; Schmutz et al., 2010).

There are costs and benefits associated with allopolyploids (Comai 2005). Allopolyploids exhibit heterosis, an increased vigor compared to the diploid progenitors (Birchler et al., 2010). Heterosis can allow hybrids to out-compete their progenitor species. The masking of deleterious alleles and genome subfunctionalization are other ways that allopolyploids maybe able to outcompete their diploid progenitor species (Mayfield-Jones et al., 2013; Madlung, 2013; Comai 2005). In addition, the formation of allopolyploids disrupts selfincompatibility mechanisms that prevent self-pollination; this disruption results in organisms that are able to undergo asexual reproduction. This is advantageous, because when allopolyploid species are formed they are often reproductively 
isolated and self-compatibility provides a way to propagate the allopolyploid genome (Comai 2005).

Allopolyploidy can cause problems in meiosis resulting in genetic changes and chromosome restructuring (Comai 2005; Gaeta et al. 2010). Two or more different genomes (subgenomes), each with a complete set of homologous chromosomes, reside inside the same nucleus. The chromosomes from one subgenome are considered homeologous to chromosomes in another subgenome (Figure 1) (Gaeta et al., 2010; Webber et al., 2004). In a diploid genome, homologs pair-up to form bivalents (Figure 2), exchange genetic material through crossovers, and segregate to create gametes with a haploid set of chromosomes from each diploid progenitor. However, in allopolyploids there is no way to ensure equal segregation when homoeologs pair and when they do they can form bivalents, trivalents, tetravalents and higher-order groupings (Figure 2). Crossovers between these groupings segregate to create aneuploid gametes and result in chromosomes with homeologous rearrangements. 


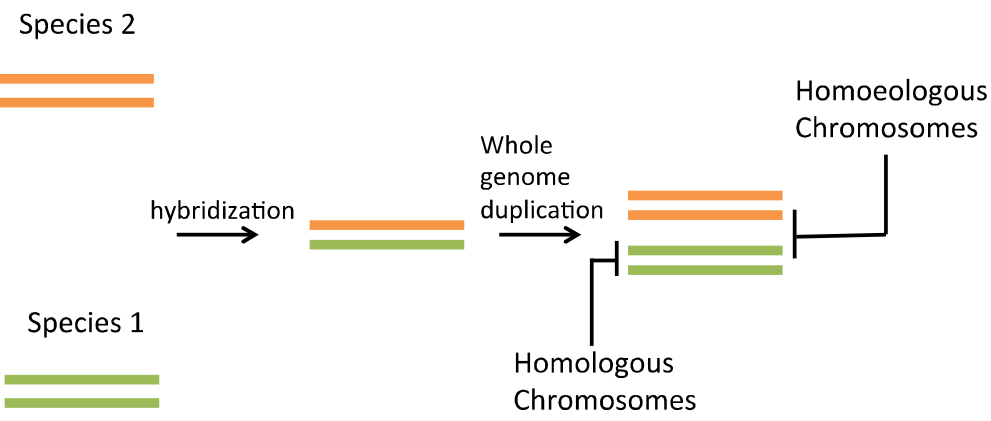

with a set of

yoes whole

genome duplication and ends up with twice as many sets of chromosomes compared to the progenitor species.

Homoeologous pairing between the sub-genomes of the progenitor species during meiosis can result in chromosome rearrangements and nondisjunction events that lower fertility (Lim et al. 2008; Gaeta et al. 2010). The offspring produced may be aneuploid or have a different ratio of chromosomes than the parent; this is called a chromosome dosage change. Dosage changes can involve duplication or deletion of whole chromosomes, or as homoeologous non-reciprocal transpositions (HNRTs); (Figure 3); (Gaeta et al. 2007). HNRTs occur when homoeologous chromosomes form crossovers during meiosis and then during strand repair the homeolog is used as a template for repair instead of the homolog (Gaeta et al. 2010). 


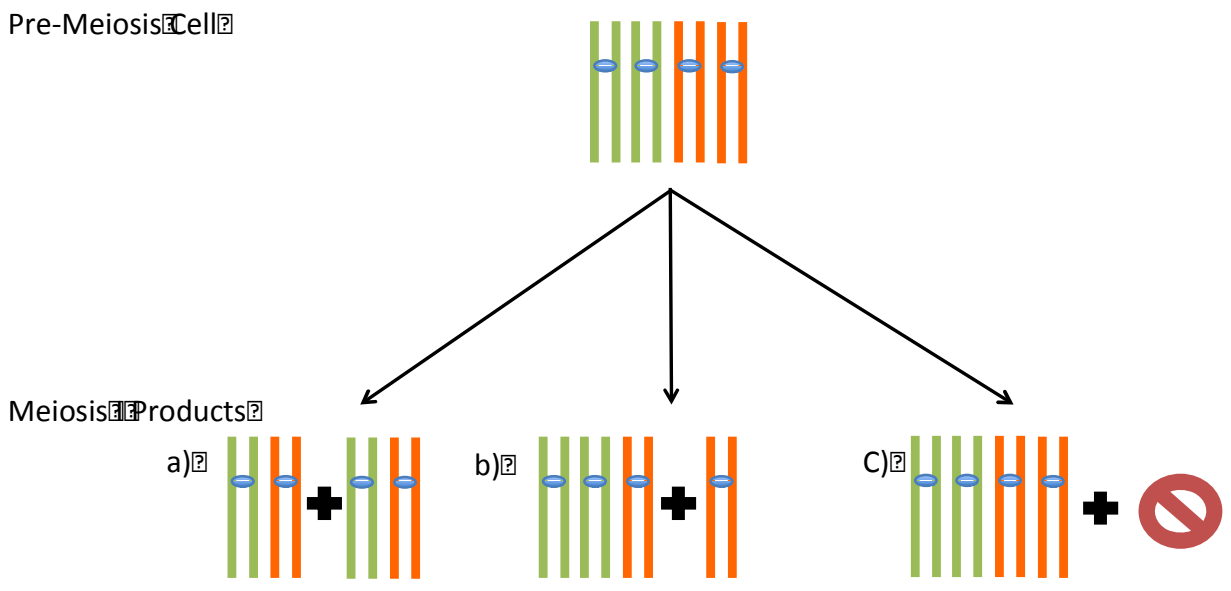

Figure 2. Possible Gametes Produced Through Cross-Overs In Tetraploids During Meiosis I.

During meiosis I homologous chromosomes cross over to form a bivalent (also known as a tetrad) which produces two diploid gametes (a). In allopolyploids homologous pairing is most common, but homeologous pairing can occur at a low rate and these homeologous pairing can lead to cross-overs to form trivalents which will produce a triploid gamete and haploid gamete (b), tetravalents which will produce a tetraploid gamete and a gamete with no chromosomes (c), or other combinations of mulitvalents. 


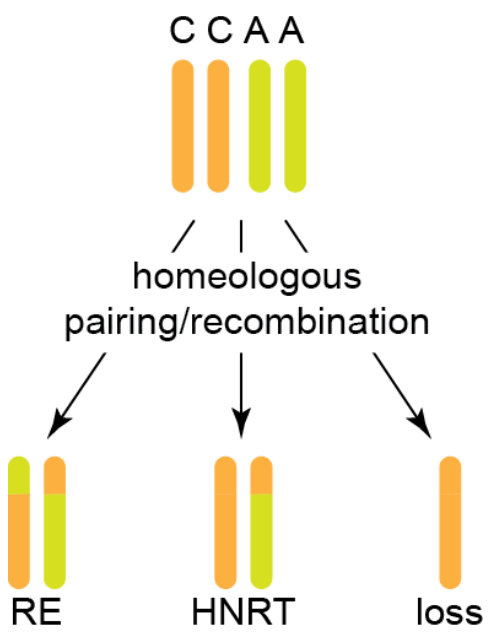

Figure 3. Different Types Of Chromosomal Rearrangements That Can Arise From Pairing Between Homeologous Chromosomes.

Brassica napus as a Model Organism

Brassica napus is a good model for studying allopolyploidy. It is an allotetraploid that formed 20mya from the hybridization of Brassica rapa and Brassica oleracea (Yang et al., 1999). Wild B. napus behaves genetically as a diploid due to a process called rediploidization (Wolfe, 2001). During rediploidization a polyploid undergoes chromosomal restructuring such that homologous pairing is promoted and homeologous pairing is suppressed. Thus, faithful pairing is restored and no further challenges to fertility are seen (RennyByfield et al., 2013).

One way to study allopolyploids is to recreate the hybridization event to generate "resynthesized" plant lines. Resynthesized B. napus plants are created by hybridizing double-haploid B. rapa and B. oleracea together (Geata et al., 2007; Lukens et al. 2006). Double haploids are most commonly created by 
chemically inducing chromosome doubling in a haploid seed or seedling (Choe et al., 2012; Dang et al., 2012). Using double haploid B. rapa and B. oleracea as progenitor parents simplifies genetic analysis by ensuring that parents are homozygous at every loci. Crossing the double haploid $B$. rapa and $B$. napus plants results in an F1 generation that is effectively a resynthesized $B$. napus. Resynthesizing hybrids in the lab allows researchers to observe the genetic restructuring following hybridization and genome duplication.

\section{Approaches To Study Chromosome Rearrangements}

Previous studies of resynthesized $B$. napus have analyzed the genetic variation of plants from distinct lineages at the same generation (i.e. 50 distinct $\mathrm{S}_{0}$ or $\mathrm{S}_{5}$ plants are compared). These studies have effectively sampled the types of genetic changes experienced by resynthesized $B$. napus plants. The driver for most genetic changes is the high degree of synteny between the $A$ and $C$ genomes (Parkin et al., 2005; Inguez et al., 2008). Homoeologous pairing and recombination during meiosis in $B$. napus can shuffle the genome producing chromosomes bearing both A and C loci (Nicolas et al., 2009; Szadkowski et al., 2010); (Figure 3). The resulting gametes are expected to vary from the expected CC:AA dosage at some loci (e.g. CCC:A and CCCC dosages); (Lukens et al., 2006; Gatea et al., 2007; Gaeta et al., 2010). The application of fluorescence insitu hybridization (FISH) to $B$. napus has revealed both reciprocal exchange (RE) between homoeologs, and non-reciprocal homoeologous transpositions (HNRT) in which a chromosome arm from one progenitor genome is replaced by one 
from the other (Xiong et al., 2011a). Another common change involves loss of an entire $\mathrm{A}$ or $\mathrm{C}$ chromosome. Chromosome loss can be balanced $(C C: A A \rightarrow C C C: A)$ or unbalanced $(C C: A A \rightarrow C C: A)$ depending on whether or not the loss of a chromosome is accompanied by the gain of a homoeolog. Interestingly, while chromosome loss during meiosis appears to be frequent, levels of aneuploidy among resynthesized $B$. napus are fairly low with most plants having 36-42 chromosomes (Xiong et al., 2011b). This observation suggests that balanced dosage changes tend to predominate. RE, HNRT and dosage change are not unique to $B$. napus and are observed in other allopolyploid species (Salmon et al., 2010; Tate et al., 2010).

However, while some rearrangements may "stabilize" the allopolyploid genome, others may disrupt stability. Gaeta and Pires (2010) describe the accumulation of chromosomal rearrangements in allopolyploids as a "ratchet-like mechanism" in which dosage changes caused by chromosomal rearrangements induce more chromosomal rearrangements in later rounds of meiosis (Figure 4). Recombination between homeologs during meiosis produces chromosomes carrying material from both subgenomes. The presence of these rearranged chromosomes increases the likelihood that non-homologous chromosomes will pair during meiosis in the next generation. 


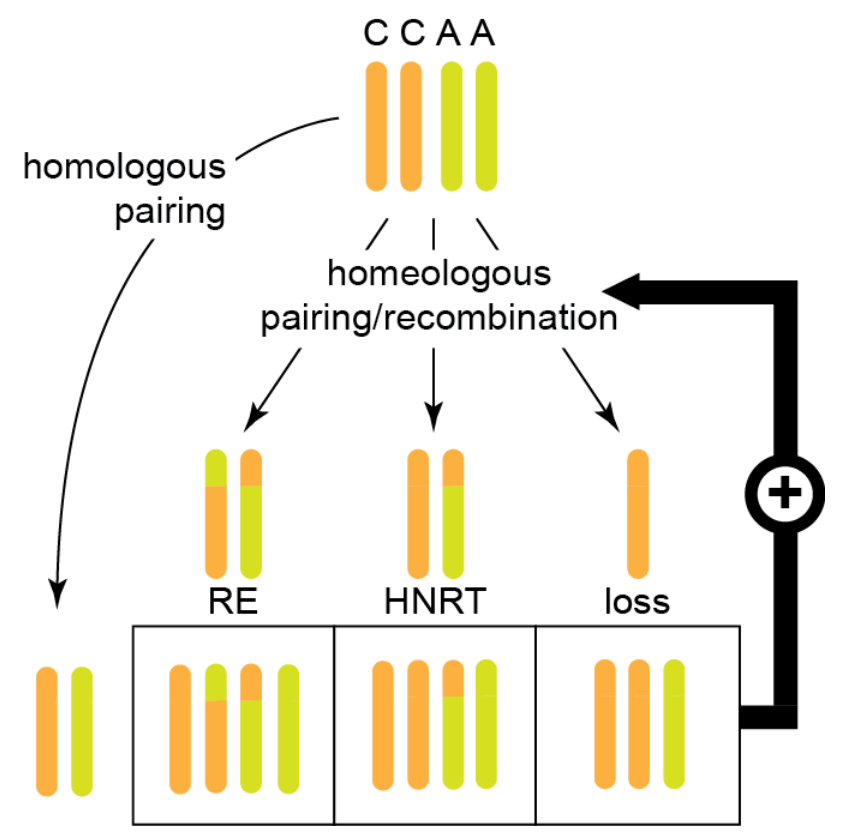

Figure 4. "The Polyploid Ratchet".

When an allopolyploid undergoes meiosis either homologous pairing, pairing between chromosomes of the same subgenome, or homeologous pairing, pairing between chromosomes of different subgenomes. Homoeologous pairing can lead to chromosomal rearrangements, which in subsequent rounds of meiosis, can lead to even more chromosomal rearrangements. These chromosomal rearrangements accumulate such that later generations will have more chromosomal rearrangements than earlier generations.

Genetic markers for allopolyploid studies are ideally represent one locus per sub genome and are able to distinguish between homoeologous sequences at homeologous loci. In allopolyploid plants there are many duplicated regions of DNA and in order for a genetic marker to work for dosage counting, it must occur 
at a single locus per subgenome, otherwise dosage measurements will be inflated (Salentijn et al., 2009; Deschamps et al., 2010).

Chromosome rearrangements can be detected using several techniques, each of which has advantages and disadvantages. One way to visualize chromosome rearrangements is with fluorescent in-situ hybridization (FISH), which uses fluorescently labeled probes for each chromosome allowing the researcher to identify where homoeologous regions are located in the allopolyploid and what their dosages are. This method creates karyotype images that examine the whole genome and is dosage sensitive. However, FISH cannot detect inheritance patterns, is labor intensive, has a low yield-to-effort ratio, and is very expensive (Lim et al., 2008). Another technique that utilizes a genomic probe is Southern Blot, where specific sequences of DNA are hybridized to a membrane and then visualized in an image. It is able to detect differences between homoeologous chromosomes, and reveal dosage changes. However, it is expensive and requires radiolabeled nucleotides (Lukens et al., 2006; Lange et al., 2011). A third common technique is polymerase chain reaction (PCR), which uses DNA synthesis to amplify a target region of DNA. Since PCR works by using two primers that mark the beginning and end of the desired DNA region, one marker can only be used to distinguish between homoeologous loci when DNA region in each homoeologous subgenome has a size polymorphism (a large size difference is greater than 50bp) (Lukens et al., 2006). PCR is quick, inexpensive, and easy, however it cannot distinguish dosage changes. 
A single nucleotide difference in sequence between two strands of otherwise syntenous DNA is called single nucleotide polymorphisms (SNP) (Figure 5). Common techniques for evaluating SNPs are PCR in conjunction with restriction digest or pyrosequencing. PCR in conjunction with restriction digest uses SNPs that occur in restriction sites. The SNP must fall within a restriction enzyme site such that enzyme will cleave one subgenome and not the other (Figure 5). Alleles can be detected by looking at the size of PCR products after enzyme digest. This technique is simple, however it lacks sensitivity of chromosomal dosages (Agarwa et al., 2007).

\section{ACCACGACATCATCTACG? ACCACGACATAATCTACG?}

\section{Figure 5. SNPs.}

The red nucleotide highlights a single nucleotide polymorphism (SNP). Sequence in blue flanking the SNP is a restriction enzyme site. The sequence in green is the homoeologous region with a restriction site that cannot be cleaved by the corresponding enzyme.

Pyrosequencing is a sequencing-by-synthesis method that uses fluorescence to track nucleotide incorporation into a newly synthesized cDNA. Whenever a nucleotide is incorporated a flash of light is detected with intensity proportional to the number of nucleotides incorporated at one time. Following 
light produced during a series of nucleotide dispensations thus determines the sequence of the DNA strand (Figure 6);(Qiagen 2009). When a short region of DNA that contains a SNP is analyzed, there will be a difference in light intensity produced at the SNP position. The light intensity for each of the mismatched nucleotides of the SNP indicates their ratio in the genome, which is representative of the subgenome ratio at that locus. The ability to accurately track the sequence of a DNA strand and determine the ratio of nucleotides at a specific location makes pyrosequencing sensitive to the chromosome dosages (Figure 6). After the initial costs of acquiring a pyrosequencing machine, pyrosequencing is fast, easy, and relatively inexpensive (Rickert et al., 2002; Vignal et al., 2002; Salentijin et al., 2009; Deschamps et al., 2010). 


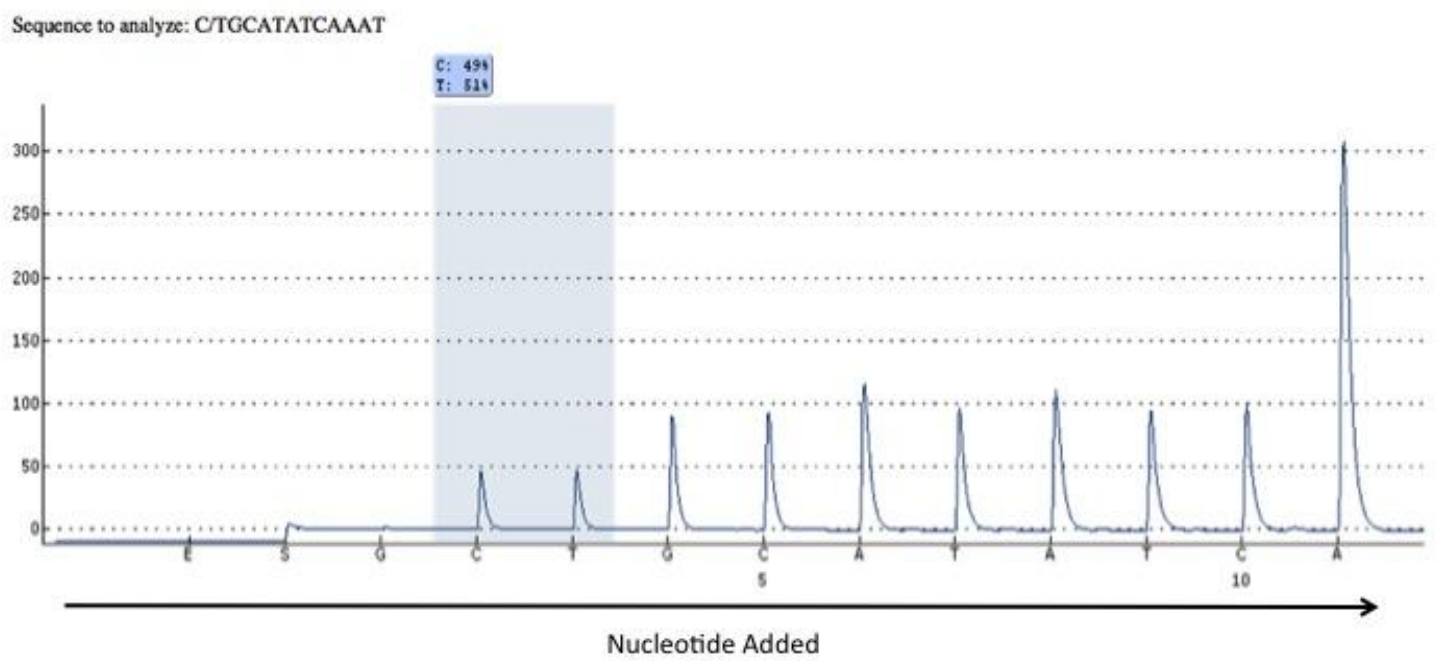

Figure 6. Example Of A Pyrosequence Across A SNP-Containing Region. The sequence analyzed here is (C/T)GCATATCAAA (the SNP is in the first position and is either a $\mathrm{C}$ or a $\mathrm{T}$ ). The $\mathrm{Y}$-axis represents fluorescence intensity. On the pyrogram the fluorescence associated with the SNP nucleotides are highlighted in blue. The DNA sample analyzed is a 1:1 mixture of DNA from Brassica rapa (T nucleotide) and Brassica oleracea ( $\mathrm{C}$ nucleotide). The fluorescence detected when either SNP nucleotide is incorporated is roughly proportional to template DNA ratio. A nucleotide present in both genomes (e.g. the G immediately following the SNP) emits a fluorescence roughly twice the intensity of that emitted from either SNP nucleotide.

Previous Research

Previously DNA was collected from a parent Brassica napus plant $\left(\mathrm{S}_{1}\right.$ generation) and 39 offspring produced by self-pollination ( $\mathrm{S}_{2}$ generation). One SNP located in the FLC3 gene at the tip of chromosome three was characterized and used to analyze the $S_{1}$ plant. The resulting ratio of subgenomes present at 
the FLC3 locus was three B. oleracea chromosomes: one B. rapa chromosome. Analysis of the $39 \mathrm{~S}_{2}$ plants by PCR analysis using this SNP identified six $\mathrm{S}_{2}$ plants missing the B. rapa chromosome at that locus. Additional SNPs at multiple places along chromosome three will need to be identified to determine if HNRTs occurred in between the S1 and the S2 generations. Because PCR and RFLP analysis is dosage insensitive, we will use pyrosequencing to analyze these SNPs in the plants already characterized (Wang and Himelblau, unpublished).

The hypothesis of this study was that if a parent has chromosome rearrangements at a locus, the offspring will have more chromosomal rearrangements surrounding and including that locus. 


\section{CHAPTER II: METHODS}

\section{Greenhouse}

Hybridization of Brassica oleracea (TO1000, egg donor; C-genome) and Brassica rapa (IMB218; pollen donor; A-genome) produced resynthesized $B$. napus allopolyploid plants (CCAA) as described previously by Lukens et al. (2006). Brassica rapa and B. oleracea used to generate B. napus are doubled haploids, and thus are expected to be homozygous at every locus. The CA hybrids produced in the original crosses were treated with colchicine to induce genome doubling that produce resulting in the first allopolyploid generation $\left(\mathrm{S}_{0}\right)$. Lineages were propagated for 12 generations by self pollination as described in Gaeta et al. (2007). Two such lineages were selected for analysis. For each lineage a single S1, S6 and S11 plant was grown and DNA was extracted. These plants were self-pollinated. Approximately 32 S2, S6, and S12 plants were grown from each of the original 6 parents. All six parent plants and three progeny pools were selected for dosage-sensitive marker analysis.

\section{DNA Extraction and Sequencing}

Genomic DNA was extracted using the Qiagen Plant Mini-Prep: DNA Extraction Kit (Qiagen; Valencia, Ca, USA). Three polymorphic markers were developed for the top (FLC3 locus), middle (the Bra012552 locus), and bottom (the Bra017743 locus) of the A3/C3 homeolog pair. DNA fragments were amplified using the following conditions: $1.0 \mathrm{U}$ of GoTaq Flexi DNA Polymerase 
(Promega Corporation; Madison, WI, USA), 25 mM MgCl, $5 X$ Green GoTaq

Flexi Buffer, $2.5 \mu \mathrm{M}$ of dNTPs, $10 \mu \mathrm{M}$ each of forward and reverse primers, $10 \mathrm{ng}$ of plant DNA, and $\mathrm{dH}_{2} \mathrm{O}$ to final volume of $20 \mu \mathrm{l}$. PCR conditions for $F L C 3$ and 12.138 were as follows: $95^{\circ} \mathrm{C}$ for $30 \mathrm{sec}$; eight cycles of $94^{\circ} \mathrm{C}$ for $30 \mathrm{sec}, 50^{\circ} \mathrm{C}$ for $30 \mathrm{sec}, 72^{\circ} \mathrm{C}$ for $50 \mathrm{sec} ; 22$ cycles of $89^{\circ} \mathrm{C}$ for $30 \mathrm{sec}, 50^{\circ} \mathrm{C}$ for $30 \mathrm{sec}, 72^{\circ} \mathrm{C}$ for $50 \mathrm{sec} ; 72^{\circ} \mathrm{C}$ for $30 \mathrm{sec}$. PCR conditions for 2.2 were as follows: $95^{\circ} \mathrm{C}$ for $30 \mathrm{sec}$; eight cycles of $94^{\circ} \mathrm{C}$ for $30 \mathrm{sec}, 55^{\circ} \mathrm{C}$ for $30 \mathrm{sec}, 72^{\circ} \mathrm{C}$ for $50 \mathrm{sec} ; 22$ cycles of $89^{\circ} \mathrm{C}$ for $30 \mathrm{sec}, 55^{\circ} \mathrm{C}$ for $30 \mathrm{sec}, 72^{\circ} \mathrm{C}$ for $50 \mathrm{sec} ; 72^{\circ} \mathrm{C}$ for $30 \mathrm{sec}$.

\section{Search for Non-Orthologous or-Paralogous genes}

The varieties of $B$. rapa and $B$. oleracea used in this study are not sequenced or publicly available, thus the published genome of closely related varieties were used to help identify candidate SNPs. Known and hypothesized genes on chromosome $A 3$ of $B$. rapa and $B$. oleracea were identified, because they are more likely to contain a SNP surrounded stretches of homologous DNA. Potential genes were selected from either a previously published $B$. napus gene map (Parkins et al., 2005) or selected using the Genome Browser tool from the Brassica Rapa Data Base (BRAD) (Wang, X., et al., 2011). B. rapa sequences identified in this way were used to search an unpublished draft of the $B$. oleracea genome (Pires, unpublished). Using the Basic Alignment Search Tool (BLAST), potential $B$. oleracea homeologs on the $\mathrm{C} 3$ chromosome were identified. If a $B$. rapa sequence had significant matches on more than one $B$. oleracea chromosome or in more than one place on a chromosome, it was discarded. If a 
gene had only one significant match, the genetic marker sequence was saved as a FASTA file and assigned a code for later use.

\section{Amplification and Sequencing of Candidate Markers}

PCR primers were developed to amplify the potential gene region using the FASTA files from BRAD (Wang, X., et al., 2011) and Primer3 (Rozen et al., 2000) (Appendix I). Primers were tested on 10ng of DNA from B. rapa, $B$. oleracea, and a synthetic hybrid composed of a 1:1 mixture of $B$. rapa and $B$. oleracea DNA. Initial PCR mixture used was: $1.0 \mathrm{U}$ of GoTaq Flexi DNA Polymerase (Promega Corporation; Madison, WI, USA), 25 mM MgCl, $5 X$ Green GoTaq Flexi Buffer, $2.5 \mu \mathrm{M}$ of dNTPs, $10 \mu \mathrm{M}$ each of forward and reverse primers, $10 \mathrm{ng}$ of plant DNA, and $\mathrm{dH}_{2} \mathrm{O}$ to final volume of $20 \mu \mathrm{l}$. A thermocycler program with an annealing temperature gradient was used: $94^{\circ} \mathrm{C}$ for 3 minutes, 8 cycles of $94^{\circ} \mathrm{C}$ for 30 seconds, $50-60^{\circ} \mathrm{C}$ for 30 seconds, $72^{\circ} \mathrm{C}$ for 50 seconds, 27 cycles of $89^{\circ} \mathrm{C}$ for 30 seconds, $50-60^{\circ} \mathrm{C}$ for 30 seconds, $72^{\circ} \mathrm{C}$ for 50 seconds, then $72{ }^{\circ} \mathrm{C}$ for 3 minutes. DNA fragments were separated using $6.0 \%$ polyacrylamide gel exposed to $300 \mathrm{~V}$ for 1.5 hours. Fragments were visualized using ethidium bromide and a UV transilluminator and analyzed using Quantity One 4.6.3 Gel Doc EQ (BioRad, Hercules, CA, USA). The PCR protocol was optimized for primers that successively amplified in both parents.

Amplified fragments were purified using Wizard SV Gel and PCR CleanUp system (Promega Inc., Madison, WI, USA). Fragments were sequenced by 
Sanger sequencing using both the forward and reverse primers (GeneWiz Inc., Plainfield, NJ, USA)

Identification and Validation of SNPS

$B$. rapa and $B$. oleracea sequences were aligned using the PairwiseBLAST to locate SNPs. The following criteria were used to identify appropriate SNPs for pyrosequencing: SNPs that were flanked by at least 30 bp of identical sequence, SNPs did not contain an adenine, and the flanking region did not contain more than three identical nucleotides in a row (e.g. AAA, TTTTT, etc.).

Derived Cleaved Amplified Polymorphism (dCAP) markers were developed such that one version of the SNP was artificially turned into a restriction digest site (Appendix I). Following amplification, SNPs were digested with Hpal (testing the FLC3 locus), Sall (testing the Bra012552 locus), or EcoRV (testing the Bra017743 locus) then visualized on a gel as described above. Once SNPs were verified by dCAP analysis, pyrosequencing primers and assays were designed around the SNP.

Pyrosequencing

PCR and sequencing primers for pyrosequencing were designed using PyroMark Assay Design Software 2.0 (Qiagen, USA)(Table \#). Pyrosequencing assay was developed by the PyroMark Q24 Analysis Software (Qiagen, USA) and performed on the PyroMark Q24 (Qiagen, USA). 
The FLC3 locus PCR mixture final concentrations used were: $0.06 \mathrm{U}$ of GoTaq Flexi DNA Polymerase (Promega Corporation; Madison, WI, USA), 2.2 $\mathrm{mM} \mathrm{MgCl} 2,1 \mathrm{X}$ Green GoTaq Flexi Buffer, $1.3 \mu \mathrm{M}$ dNTPs, $0.2 \mu \mathrm{M}$ each of forward and reverse primers, $10 \mathrm{ng}$ of plant DNA, and $\mathrm{dH}_{2} \mathrm{O}$ to final volume of $25 \mu \mathrm{l}$. The Bra012552 locus PCR mixture final concentrations used were: $0.06 \mathrm{U}$ of GoTaq Flexi DNA Polymerase (Promega Corporation; Madison, WI, USA), 2.2 mM $\mathrm{MgCl}_{2}, 1 \mathrm{X}$ Green GoTaq Flexi Buffer, $0.8 \mu \mathrm{M}$ dNTPs, $0.2 \mu \mathrm{M}$ each of forward and reverse primers, $10 \mathrm{ng}$ of plant DNA, and $\mathrm{dH}_{2} \mathrm{O}$ to final volume of $25 \mu \mathrm{l}$. The Bra017743 locus PCR mixture final concentrations used were: $0.06 \mathrm{U}$ of GoTaq Flexi DNA Polymerase (Promega Corporation; Madison, WI, USA), 2.2 mM $\mathrm{MgCl}_{2}, 1 \mathrm{X}$ Green GoTaq Flexi Buffer, $0.8 \mu \mathrm{M}$ dNTPs, 1X of Qiagen custom oligos, $10 \mathrm{ng}$ of plant DNA, and $\mathrm{dH}_{2} \mathrm{O}$ to final volume of $25 \mu \mathrm{l}$.

PCR thermocycler settings for the SNP at the Bra017743 locus and the SNP at the Bra012552 locus: 1) $95^{\circ} \mathrm{C}$ for $5: 00 \mathrm{~min}$, 2) $94^{\circ} \mathrm{C}$ for $\left.30 \mathrm{~s}, 3\right) 56^{\circ} \mathrm{C}$ for $45 \mathrm{~s}$, 4) $72^{\circ} \mathrm{C}$ for $50 \mathrm{~s}$, 5) Repeat $\left.2-435 \mathrm{x}, 6\right) 72^{\circ} \mathrm{C}$ for $\left.3: 00 \mathrm{~min}, 7\right) 4^{\circ} \mathrm{C}$ for $\bullet \min$. Pyrosequencing Binding Mix for the FLC3, Bra012552, and Bra017743 loci was $40 \mu$ l Binding Buffer, $18 \mu \mathrm{l}$ Nanopure water, $2 \mu$ l Streptavidin Beads. The assay buffer mix for FLC3 and Bra012552: $25 \mu$ Annealing Buffer and 0.75 $\mu l$ 10 $\mu$ M Sequencing Primer. The assay Buffer Mix for the Bra017743 SNP: 22.5 $\mu \mathrm{l}$ Annealing Buffer and $2.5 \mu \mathrm{l}$ 10x Sequencing Primer.

Statistical Analysis of Pyrosequencing Data 
Most samples were assayed twice, some were able to be assayed only once. In cases of multiple assays, the results of each individual were averaged to deliver a single value. A discriminant analysis was run on each genetic marker using dosage controls created by mixing $B$. rapa and B. oleracea DNA in specific rations (AAA:C, AA:C, A:C, A:CC, A:CCC) and parental DNA (A only, $C$ only). Dosage controls comprised training groups for discriminant analysis for each marker. Only predicted ratio designations that had a probability of a correct designation larger than 0.80 were retained for further analysis. Chi-squared analysis was used to compare the observed data with the predictions of Mendelian Models.

All statistics were performed on JMP 10 statistical software (SAS Institute Inc., 2012). 


\section{CHAPTER III: RESULTS}

\section{Dosage Changes At Loci Along The A3:C3 Homeologs}

To determine rates of homeologous rearrangements on an acrocentric chromosome in $B$. napus, dosage-sensitive pyrosequencing markers were developed at three loci along the A3:C3 homeologs (FLC3, Bra012552, Bra017743). Resynthesized B. napus plants with no rearrangements between homeologs are expected to have the dosage AA:CC at all loci. HNRT is expected to alter dosage at the affected loci. Dosage changes can be balanced (e.g, AA:CC to A:CCC) or unbalanced (e.g. AA:CC to A:CC).

Populations of resynthesized $B$. napus were generated by crossing $B$. rapa (A genome) and $B$. oleracea ( $\mathrm{C}$ genome) and colchicine-treating the hybrid progeny to induce genome doubling (Gaeta, 2007; Lukens, 2006). To create plants with two copies of each A- and C-subgenomes, the A and C genome parents are doubled-haploids and are therefore expected to be homozygous at all loci. Two resynthesized B. napus plants were self-pollinated for 11 generations.

Six independent plants from two different lineages were genotype with the SNP markers at all three loci (Figure 7). There were two S1, two S6, and two S11 plants. Four of those six plants were AA:CC at all loci tested (Table A, Figure B). The other two plants were AA:CC at the Bra012552 and Bra017743 loci, but had changes at the FLC3 locus. One plant (parent \#3) was a AA:C at the FLC3 locus. Another plant (parent \#2) had a complete loss of the C genome at the FLC3 locus, indicating a deletion likely occurred. These two parents presented an 
opportunity to observe how dosage changes segregate during meiosis. A third plant (parent \#1) was AA:CC at all loci tested, indicating that no dosage changes had occurred, and thus we were able to use it as a comparison the parents that had dosage changes (Figure 7). These plants were self-pollinated and about 35 progeny were grown from each. Parent \#1 was self-pollinated to produce progeny population \#1, parent \#2 was self-pollinated to produce progeny population \#2, and parent \#3 was self-pollinated to produce progeny population \#3. DNA was extracted from the progeny. The progeny were then genotyped at the same three loci to determine how parental genotype influenced the inheritance by the progeny. 

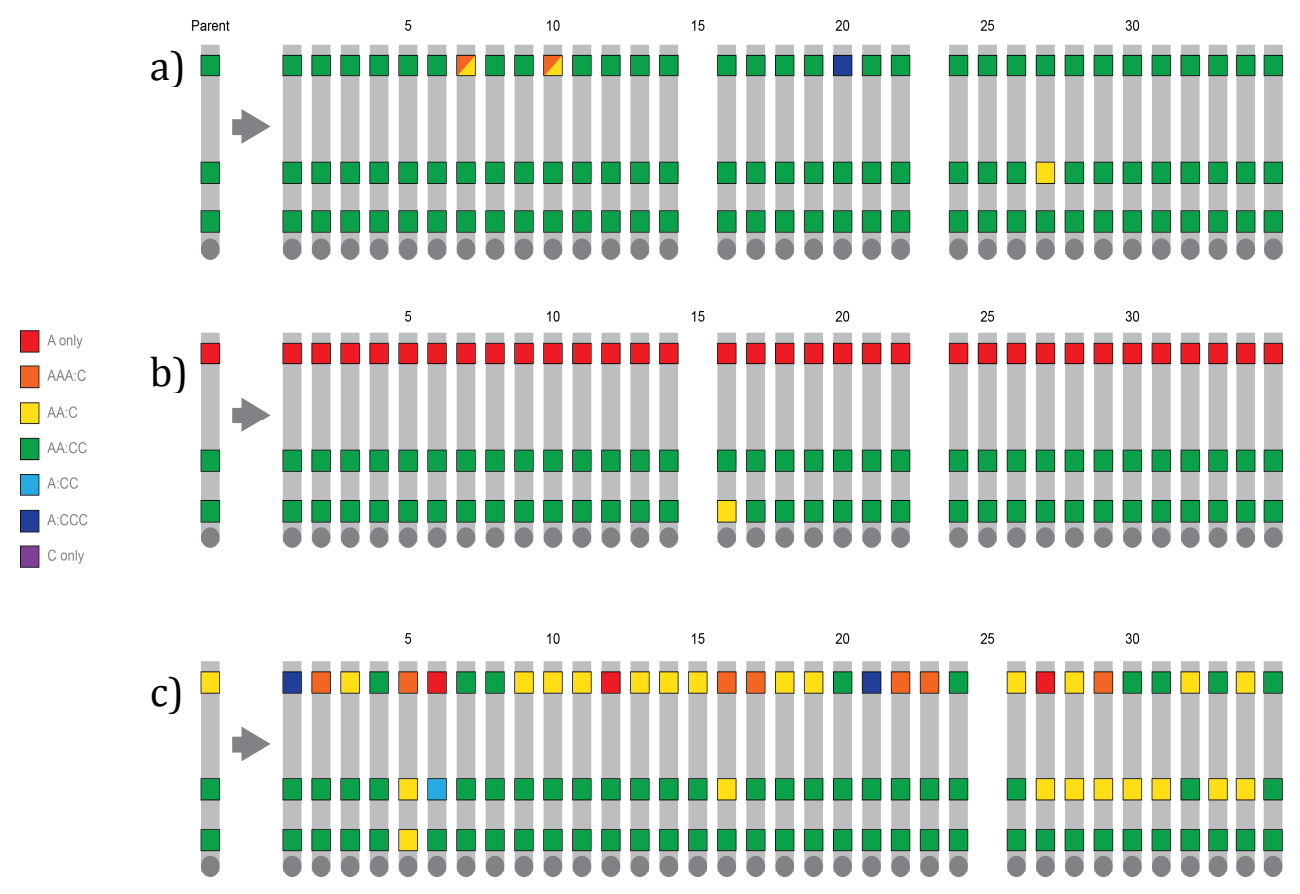

Figure 7. Chromosome Diagrams Of Parents And Their Progeny.

Parent \#1 (AA:CC at all loci) was self-pollinated to produce population \#1 (a). Parent \#2 (A only at the FLC3 locus, AA:CC at the Bra012552 and Bra017743 loci) was self-pollinated to produce population \#2 (b). Parent \#3 (AA:C at the FLC3 locus, and AA:CC at the Bra012552 and Bra017743 loci) was selfpollinated to produce population \#3 (c). The circle at the bottom of the chromosome represents the centromere and the squares represent the loci tested. "A" represents the $B$. rapa subgenome and " $C$ " represents the $B$. oleracea subgenome. 
Frequency Of Novel Rearrangements

To investigate the frequency of novel rearrangements (i.e. homeologous exchange during meiosis in a parent plant) parent \#1 was self-pollinated and the progeny, population \#1, were analyzed at the three loci. In population \#1 there were four individuals with dosage changes (Figure 7). One individual was A:CCC at the FLC3 locus, two were either AA:C or AAA:C at the FLC3 locus, and another individual was AA:C at the Bra012552 locus. No dosage changes were observed at the Bra017743 locus. Since these individuals only have a dosage change at one locus, a HNRT likely occurred during meiosis in the parent.

Parent \#2 had complete loss of the C genome at the FLC3 locus and was AA:CC at the other loci tested. Most offspring of parent \#2, population \#2, had the same genotype as parent \#2, only one of the offspring was missing the $\mathrm{C}$ genome at the FLC3 locus, AA:CC at the Bra012552 locus, and AA:C at the Bra017743 locus. This dosage change occurs close to the centromere. Deletion at the distal end of the chromosome appears to have had little effect on homeologous pairing or exchange elsewhere in the chromosome.

\section{Segregation Of Rearrangements Present In The Parent}

Parent \#3 has a dosage of $A A: C$ at the FLC3 locus and AA:CC at the Bra012552 and Bra017743 loci. The FLC3 locus segregated into the offspring generation such that three offspring had a complete loss of the $\mathrm{C}$ genome, nine were $A A: C C$, thirteen were $A A: C$, seven were $A A A: C$, and two offspring were 
A:CCC at the FLC3 locus. Novel changes at other loci were also observed. At the Bra012552 locus, seven were AA:C, and one was A:CC. At the Bra017743 locus one individual was AA:C (Figure 7). 


\section{CHAPTER IV: DISCUSSION}

\section{Allopolyploidy in Brassica napus}

Allopolyploid genomes form by hybridization of two diploid genomes followed by duplication. Thus an allopolyploid is expected to have an equal dosage of loci from each subgenome. B. napus is allopolyploid of B. rapa (Asubgenome) and B. oleracea (C-subgenome) and is therefore expected to be AA:CC at all loci. Homeologous pairing between the A- and C-subgenomes can lead to dosage changes. If a plant with a dosage change at a particular locus self-pollinates, the dosage change will segregate in the progeny. In addition, the presence of the dosage change in the parent may increase the likely hood of additional homeologous pairing and exchange at other loci. Here plants with and without dosage changes were self-pollinated and the progeny genotype was determined. Both novel dosage changes arising during meiosis in the parent and segregation patterns in the progeny were observed.

Inheritance of Homeologous Exchanges

Parent \#1 most likely has two A chromosomes and two $\mathrm{C}$ chromosomes. Since parent \#1 has no dosage changes, it is unlikely that there would be dosage changes in the offspring. Three out of 33 of the progeny were identified with a different dosage from the parent at the FLC3 locus and one individual with a dosage different at the Bra012552 locus. Of the loci tested, the FLC3 locus is the most distal from the A3/C3 centromere. Since each progeny plant is the result of 
two independent meiosis events, we concluded that in a parent three of 66 meioses involved recombination between the $\mathrm{A} 3$ and $\mathrm{C} 3$ genome across an interval containing the $F L C 3$ locus. In many plants recombination of chromosomes increases in regions further away from the centromere (Drouaud 2006).

It is straightforward to envision how balanced dosage changes (i.e. AA:CC changing to $A: C C C$ ) come about. These result from crossing over between homeologs in which roughly equivalent regions are exchanged. It is more difficult to envision a mechanism for unbalanced dosage changes (i.e. AA:CC changing to $A: C C)$ especially in cases where other loci along the homeologs have maintained normal AA:CC dosage. We have identified two such cases. Presumably these individuals have two A3 and two C3 chromosomes (explaining the normal dosage observed at 2 or 3 loci) but have a deletion of a locus from one of the chromosomes producing a AA:C or A:CC dosage at that locus only. Unequal crossing over between homologous chromosomes is known to cause segmental duplications and deletions (Szostak et al., 1980). Between homologs, unequal crossover can be initiated by imprecise pairing. We postulate that precise pairing between homeologs is unlikely particularly in more diverged regions. Therefore, it may not be unexpected to find an increased frequency of duplication and deletion in an allopolyploid genome where both imprecise homologous pairing and unequal homeolog pairing are both possibilities.

Parent \#2 has only the A genome present at the FLC3 locus and AA:CC at the other two loci, and probably has this genotype as a result of either a deletion 
of the $\mathrm{C}$ genome from the region containing the $F L C 3$ locus or a homeologous translocation. If the genotype arose due to a deletion, one would expect little interaction between the A3 and C3 homeologes, because of the lack of synteny between the homeologes in the region containing the FLC3 locus. If the genotype arose due to a homeologous translocation, then one would expect the offspring to have a higher proportion of chromosome rearrangements because of the additional synteny from the region containing the FLC3 locus. What was seen was that only one offspring in population \#2 had a genotype different from parent \#2. This individual had only the A genome at the $F L C 3$ locus, $A A: C C$ at the Bra012552 locus, and AA:C at the Bra017743 locus (Figure 7). The fact that only one out of 33 offspring had a genomic rearrangement different from the parent supports the idea that the parent's genotype arose from a deletion of the region containing the FLC3 locus from the C-genome. The FLC3 locus is at the chromosome tip furthest from the centromere and when the region containing the FLC3 locus was deleted the whole tip may have been lost. Since there were very few genomic changes in the offspring, this type of deletion does not seem to influence pairing such that homeologous recombination increases.

Frequency of Homeologous Exchange on an Acrocentric Chromosome

Parent \#3 AA:C at the FLC3 locus and AA:CC at the Bra012552 and Bra017743 loci and this genotype most likely arose due to a deletion of the 
region containing the $F L C 3$ locus from of the $\mathrm{C} 3$ chromosomes. According to Mendelian principles, one would expect this rearrangement to segregate such that approximately $50 \%$ of the offspring would be the same as the parent, $25 \%$ would be AA:CC at all loci, and the last $25 \%$ would be missing the region containing the $F L C 3$ locus from the $C$ genome entirely (Figure 8).

What we saw was that $32.4 \%$ of the offspring were the same genotype as the parent, $17 \%$ were $\mathrm{AA}: \mathrm{CC}$ at all loci, $2.9 \%$ were only $\mathrm{A}$ at the $F L C 3$ locus and AA:CC at the other loci, and $47 \%$ were genotypes not predicted by the Mendelian hypothesis. This non-mendelian segregation suggests that homeologous exchange is occurring during meiosis.

The presence of rearrangements distal to the centromere and the absence of rearrangements proximal to the centromere on $A 3 / C 3$ in our populations could be explained two ways. First, it could be a general phenomenon that homeologous pairing and recombination are suppressed nearer to the centromere. A similar pattern has been seen in the B. napus $\mathrm{C} 1-\mathrm{A} 1$ homeolog pair (Nicolas 2012). Second, the patterns of recombination observed could be highly specific to the $\mathrm{A} 3 / \mathrm{C} 3$ chromosome and not represent a general phenomenon. A3 and C3 may be more syntenous at the distal ends of the chromosome and, therefore, more likely to pair and recombine in this region. Genetic maps show relatively high levels of synteny along $A 3 / C 3$ yet the greatest synteny is found at the distal end of the chromosome (Nicolas 2012; Xiong 2011; Parkin 2005). Soon a physical map for Brassica oleracea will be available that will allow the level of synteny between A3 and C3 to be determined with certainty. 
At this point it would be premature to attribute the patterns of homeologous exchange observed for $\mathrm{A} 3 / \mathrm{C} 3$ to a general phenomena within allopolyploids.

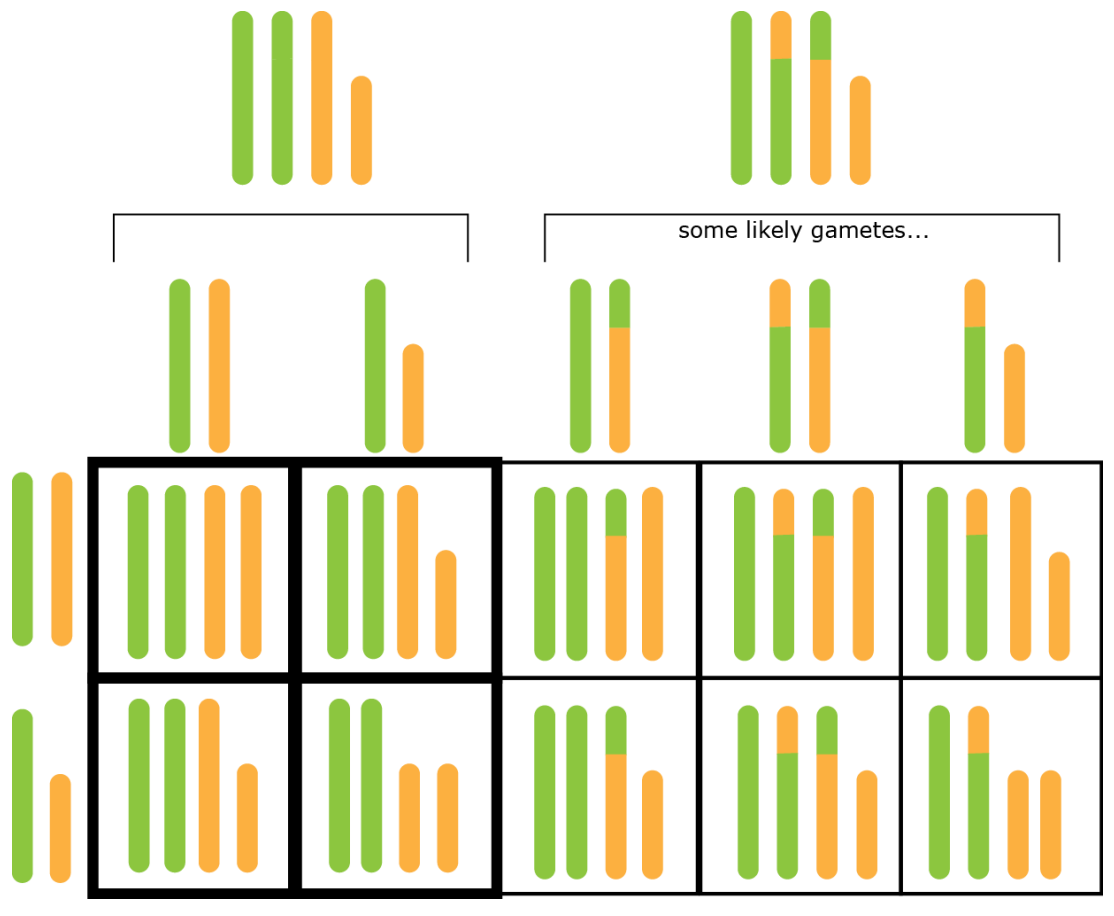

Figure 8. Mendelian and Non-Mendelian Segregation for Parent \#3.

If parent \#3's dosage is the result of a deletion at the tip of one of the chromosomes and the homeologs segregate independently, one expects a 1:2:1 ratio of the deletion in the progeny (bolded squares). If homeologous pairing that results in chromosome rearrangements in the gametes does occur, one expects more rearrangements than those predicted by independent assortment.

\section{CHAPTER V: CONCLUSION}

This approach has provided insights into the frequency with which homeologous pairing and exchange occur during meiosis in an allopolyploid. In parents with no dosage changes, novel changes occurred, but at lower rates 
when compared to a parent with dosage changes. This suggests that a dosage changes in the parent may induce or contribute to chromosomal rearrangements during meiosis and thus produce more dosage changes in the offspring. Also, not just any chromosomal rearrangement leads to further rearrangements, parent \#2, the one with only the A-subgenome at the FLC3 locus, had only one offspring with a dosage change, whereas parent \#3, the one with the $A A: C$ dosage at the FLC3 locus, had many offspring with a variety of dosage changes. Regardless of what the parent dosage was, most of the changes occurred at the FLC3 locus, which suggests that chromosomal rearrangements are more likely to occur farther from the centromere than they are closer to the centromere. 


\section{REFERENCES}

Agarwal, Milee, Neeta Shrivastava, and Harish Padh. 2008. Advances in molecular marker techniques and their applications in plant sciences. Plant Cell Reports 27: 617-631.

Birchler JA, Yao H, Chudalayandi S, Vaiman D, Veitia RA (2010) Heterosis. Plant Cell 22: 2105-2112

Buggs, Richard J.A., Srikar Chamala, Wei Wu, Jennifer A. Tate, Patrick S. Schnable, Douglas E. Soltis, Pamela S. Soltis, and W. Brad Barbazuk. 2012. Rapid, Repeated, and Clustered Loss• of Duplicate Genes in Allopolyploid Plant Populations of Independent Origin. Current Biology 22:248-252.

Cheng, Feng, Terezie Mandáková, Jian Wu, Qi Xie, Martin A. Lysak, and Xiaowu Wang. 2013. Deciphering the Diploid Ancestral Genome of the Mesohexaploid Brassica rapa. The Plant Cell. 25:1541-1554.

Choe, Eunsoo, Christine Hayot Carbonero, Kelly Mulvaney, A. Lane Rayburn And Rita H. Mumm. 2012. Improving in vivo maize doubled haploid production efficiency through early detection of false positives. Plant Breeding. 131: 399-401.

Comai, Luca. 2005. The advantages and disadvantages of being polyploid. Nature Publishing Group 6:836-846.

Dang, Ngoc-Chi, Magali Munsch, •Ingrid Aulinger, Wen Renlai, and Peter Stamp. 2012. Inducer line generated double haploid seeds for combined waxy and opaque 2 grain quality in subtropical maize (Zea mays L.). Euphytica. 
183:153-160.

Deschamps, Ste' phane, and Matthew A. Campbell. 2010. Utilization of nextgeneration sequencing platforms in plant genomics and genetic variant discovery. Mol Breeding 25:553-570.

Drouaud, Jan, Christine Camilleri Pierre-Yves Bourguignon, Aurélie Canaguier, Aurélie Bérard, Daniel Vezon, Sandra Giancola, Dominique Brunel, Vincent Colot, Bernard Prum, Hadi Quesneville, and Christine Mézard Drouaud. 2006. Variation in crossing-over rates across chromosome 4 of Arabidopsis thaliana reveals the presence of meiotic recombination "hot spots". Genome Research 16: 106.

Gaeta, Robert T., J. Chris Pires, Federico Iniguez-Luy, Enrique Leon, and Thomas C. Osborn. 2007. Genomic changes in resynthesized Brassica napus and their effect on gene expression and phenotype. The Plant Cell 19:3403-3417.

Gaeta, Robert T., and J. Chris Pires. 2010. Homoeologous recombination in allopolyploids: the polyploidy ratchet. New Phytologist 186: 18-28.

Grover C. E., J. P. Gallagher, E. P. Szadkowski, M. J. Yoo, L. E. Flagel and J. F. Wendel. 2012. Homoeolog expression bias and expression level dominance in allopolyploids. New Phytologist. 196: 966-971.

Iniguez-Luy FL, Voort AV, Osborn TC (2008) Development of a set of public SSR markers derived from genomic sequence of a rapid cycling Brassica oleracea L. genotype. Theor Appl Genet 117: 997-985 
Kim ST, Sultan SE, Donoghue MJ (2008) Allopolyploid speciation in Persicaria (Polygonaceae): Insights from a low-copy nuclear region. PNAS 105: $12370-12375$

Lange, Christian, Karolin Zerulla, Sebastian Breuert, and Jörg Soppa. 2011. Gene conversion results in the equalization of genome copies in the polyploid haloarchaeon Haloferax volcanii. Molecular Microbilogy 80:3:666-677.

Lim, K. Yoong, Douglas E. Soltis, Pamela S. Soltis, Jennifer Tate, Roman Matyasek, Hana Srubarova, Ales Kovarik, J. Chris Pires, Zhiyong Xiong, and Andrew R. Leitch. 2008. Rapid Chromosome Evolution in Recently Formed Polyploids in Tragopogon (Asteraceae). PLoS ONE 3:10:e3353 113.

Lukens LN, Pires JC, Leon E, Vogelzang R, Oslach L, Osborn TC (2006) Patterns of sequence loss and cytosine methylation within a population of newly resynthesized Brassica napus allopolyploids. Plant Phys 140: 336348

Madlung, A. 2013. Polyploidy and its effect on evolutionary success: old questions revisited with new tools. Heredity. 110:99-104

Mayfield-Jones, Dustin, Jacob D. Washburn, Tatiana Arias, Patrick P. Edger, J. Chris Pires, Gavin C. Conant. 2013. Watching the grin fade: Tracing the effects of polyploidy on different evolutionary time scales. Seminars in Cell \& Developmental Biology. 24:320-331.

Mun et al.: Sequence and structure of Brassica rapa chromosome A3. Genome 
Biology 2010 11:R94.

Nicolas, Ste' phane D., Herve' Monod, Fre' de' rique Eber, Anne-Marie Che 'vre and Eric Jenczewski. 2012. Non-random distribution of extensive chromosome rearrangements in Brassica napus depends on genome organization. The Plant Journal. 70:691-703

Okzan H, Levy AA, Feldman M (2001) Allopolyploidy-Induced Rapid Genome Evolution in the Wheat (Aegilops-Triticum) Group. Plant Cell 13: 17351747

Parkin, Isobel A. P., Sigrun M. Gulden, Andrew G. Sharpe, Lewis Lukens, Martin Trick, Thomas C. Osborn, and Derek J. Lydiate. 2005. Segmental Structure of the Brassica napus Genome Based on Comparative Analysis With Arabidopsis thaliana. Genetics Society of America 171: 765-781. Pontes O, Neves N, Silva M, Lewis MS, Madlung A, Comai L, Viegas W, Pikaard CS (2004) Chromosomal locus rearrangements are a rapid response to formation of the allotetraploid Arabidopsis suecica genome. PNAS 101:181240-18245

Qiagen ${ }^{a}$. 2009. PyroMark Q24 user manual.

Qiagen ${ }^{b}$. 2009. PyroMark Q24 analysis software user guide: for use with the PyroMark Q24.

Renny-Byfield Simon, Ales Kovarik, Laura J. Kelly, Jiri Macas, Petr Novak, Mark W. Chase, Richard A. Nichols, Mahesh R. Pancholi, Marie-Angele Grandbastien and Andrew R. Leitch. 2013. Diploidization and genome 
size change in allopolyploids is associated with differential dynamics of low- and high-copy sequences. The Plant Journal. 74:829-839.

Rickert, Andreas M., Andreas Premstaller, Christiane Gebhardt, and Peter J. Oefner. 2002. Genotyping of SNPs in a Polyploid Genome by Pyrosequencing. BioTechniques 32:592-603.

Rozen S, Skaletsky H (2000) Primer3 on the WWW for general users and for biologist programmers. Methods in molecular biology (Clifton, NJ) 132: 365-386.

Salentijn, Elma M.J., Svetlana V. Goryunova, Noor Bas, Ingrid M. van der Meer, Hetty C. van den Broeck, Thomas Bastien, Luud J.W.J. Gilissen, and Marinus J.M. Smulders. 2009. Tetraploid and hexaploid wheat varieties reveal large differences in expression of alpha-gliadins from homoeologous Gli-2 loci. BMC Genomics 10:48 doi:10.1186/1471-2164$10-48$

Salmon A, Flagel LE, Ying B, Udall JA, Wendel JF (2010) Homoeologous nonreciprocal recombination in polyploid cotton. New Phyt 186: 123-134.

Schmutz J, Cannon SB, Schlueter J, Ma J, Mitros T, Nelson W, Hyten DL, Song Q, Thelen JJ, Cheng J, Xu D, Hellsten U, May GD, Yu Y, Sakurai T, Umezawa T, Bhattacharyya M, Sandhu D, Valliyodan B, Lindquis E, Pet M, Gran D, Shu S, Goodstein D, Barry K, Futrell-Grigg M, Abernath B, Du J, Tian Z, Zhu L, Gill N, Joshi T, Libault M, Sethuraman A, Zhang C, Shinozaki K, Nguyen HT, Wing RA, Cregan P Specht J, Grimwood J, Rokhsar D, Stacey G, Shoemaker RC, Jackson SA (2010), Genome 
sequence of the palaeopolyploid soybean. Nature 463: 178-183

Szostak Jack W., and Ray Wu. 1980. Unequal crossing over in the ribosomal DNA of Saccharomyces cerevisiae. Nature 284:426 - 430 .

Szadkowski E, Eber F, Huteau V, Lodé M, Coriton O, Jenczewski E, Chèvre AM (2011) Polyploid formation pathways have an impact on genetic rearrangements in resynthesized Brassica napus. New Phytol 191: 884894

Tate JA, Joshi P, Soltis KA, Soltis PA, Soltis DE (2009) On the road to diploidization? Homoeolog loss in independently formed populations of the allopolyploid Tragopogon miscellus (Asteraceae). BMC Plant Biology 9: 80-87

Vignal, Alain, Denis Milan, Magali Sancristobal, and André Eggen. 2002. A review on SNP and other types of molecular markers and their use in animal genetics. Genet. Sel. Evol. 34: 275-305.

Wang et al. 2011. A physical map of Brassica oleracea shows complexity of chromosomal changes following recursive paleopolyploidizations. BMC Genomics 12:470. http://www.biomedcentral.com/1471-2164/12/470 Wang X, Wang H, Wang J, Sun R, Wu J, et al. (2011) The genome of the mesopolyploid crop species Brassica rapa. Nature Genetics 43.

Webber, C. and C. P. Ponting. 2004. Genes and Homology. Current Biology. 14:9:R332-R333 
Wolfe K (2001) Yesterday's Polyploids and the Mystery of Diploidization. Nature Reviews Genetics 2: 333-341

Xiong, Zhiyong, Robert T. Gaeta, and Joseph Chris Pires. 2011. Homoeologous shuffling and chromosome compensation maintain genome balance in resynthesized allopolyploid Brassica napus. Proceedings of the National Academy of Science Early Edition.

Yang, Yau-Wen, Kun-Nan Lai, Pon-Yean Tai, and Wen-Hsiung Li. 1999. Rates of Nucleotide Substitution in Angiosperm Mitochondrial DNA Sequences and Dates of Divergence Between Brassica and Other Angiosperm Lineages. Journal of Mollecular Evollution 48:597-604. 


\section{APPENDIX}

Supplemental Table 1: Primer Sequences

Amplification for Sequencing

\begin{tabular}{|l|l|l|}
\hline & Forward & Reverse \\
\hline $\mathbf{2 . 2}$ & ACGGTAAAGCAGGGGACTTT & GGCCATTTGCAAGACATTTT \\
\hline $\mathbf{1 2 . 1 3 8}$ & TTCAACTCGCAGACCAAGTG & AGGGTTGACTCCACCTTCCT \\
\hline
\end{tabular}

Verification of SNPs (dCAPS)

\begin{tabular}{|l|l|l|l|}
\hline & Forward & Reverse & Enzyme \\
\hline $\mathbf{2 . 2}$ & CCTTTCGAAAGTGTGTCGA & $\begin{array}{l}\text { GTCACGATTGTGTTCCAAG } \\
\text { AAA }\end{array}$ & $\begin{array}{l}\text { Sal I } \\
\text { (cuts A) }\end{array}$ \\
\hline $\mathbf{1 2 . 1 3 8}$ & $\begin{array}{l}\text { ACGCGCCGTATCATCGACGA } \\
\text { TAT }\end{array}$ & $\begin{array}{l}\text { CTAAGTAACCGACCCCTTC } \\
\text { G }\end{array}$ & $\begin{array}{l}\text { EcoRV } \\
\text { (cuts C) }\end{array}$ \\
\hline
\end{tabular}

Pyrosequencing (initial amplification)

\begin{tabular}{|l|l|l|}
\hline & Forward & Reverse \\
\hline $\mathbf{2 . 2}$ & $\begin{array}{l}\text { GCT CTT GGA GTT TTC GTT } \\
\text { GAG AT }\end{array}$ & $\begin{array}{l}\text { CAGATACCAAGCCAGCCTCT } \\
\text { ATT } \\
\text { (5' biotin) }\end{array}$ \\
\hline $\mathbf{1 2 . 1 3 8}$ & CACGCGCCGTATCATCGA & $\begin{array}{l}\text { GTCAATGCCTTCTGGAGAAC } \\
\text { G } \\
\text { (5' biotin) }\end{array}$ \\
\hline
\end{tabular}

Pyrosequencing (sequecning)

\begin{tabular}{|l|l|l|}
\hline & Sequencing Primer & \\
\hline 2.2 & $\begin{array}{l}\text { TAA CCC TTT CGA AAG TGT } \\
\text { G }\end{array}$ & \\
\hline $\mathbf{1 2 . 1 3 8}$ & GTATCATCGACGACAC & \\
\hline
\end{tabular}

RD/DCAP Markers DCAPs Primer FLC3 
Brassica 22 cycles PCR settings: 1) $94^{\circ} \mathrm{C}$ for $3: 00 \mathrm{~min}$, 2) $94^{\circ} \mathrm{C}$ for $\left.30 \mathrm{~s}, 3\right) 50^{\circ} \mathrm{C}$ for $30 \mathrm{~s}, 4) 72^{\circ} \mathrm{C}$ for $\left.50 \mathrm{~s}, 5\right)$ Repeat $\left.2-48 \mathrm{x}, 6\right) 89^{\circ} \mathrm{C}$ for $\left.30 \mathrm{~s}, 7\right) 50^{\circ} \mathrm{C}$ for $30 \mathrm{~s}, 8$ ) $72^{\circ} \mathrm{C}$ for 50s, 9) Repeat $\left.6-822 x, 10\right) 72^{\circ} \mathrm{C}$ for $\left.3: 00 \mathrm{~min}, 11\right) 4^{\circ} \mathrm{C}$ for $\bullet$.

FLC3 DCAP RD Mixture: $5 \mu \mathrm{l}$ DNA ( $5 \mathrm{ng} / \mu \mathrm{l}), 2 \mu \mathrm{l}$ 10x Hpal buffer, $0.5 \mu \mathrm{l} \mathrm{Hpal,}$ and $12.5 \mu \mathrm{ldiH} 2 \mathrm{O}$ for a total volume of $20 \mu \mathrm{l}$. Incubate at $37^{\circ} \mathrm{C}$ for about 3 hours (2hrs minimum, overnight ok).

DCAPs 12.138 PCR setting: 1) $94^{\circ} \mathrm{C}$ for $\left.3: 00 \mathrm{~min}, 2\right) 94^{\circ} \mathrm{C}$ for $\left.30 \mathrm{~s}, 3\right) 50^{\circ} \mathrm{C}$ for $30 \mathrm{~s}, 4) 72^{\circ} \mathrm{C}$ for $\left.50 \mathrm{~s}, 5\right)$ Repeat $\left.2-48 \mathrm{x}, 6\right) 89^{\circ} \mathrm{C}$ for $\left.30 \mathrm{~s}, 7\right) 50^{\circ} \mathrm{C}$ for $\left.30 \mathrm{~s}, 8\right) 72^{\circ} \mathrm{C}$ for $50 \mathrm{~s}, 9)$ Repeat $6-827 \mathrm{x}, 10) 72^{\circ} \mathrm{C}$ for $\left.3: 00 \mathrm{~min}, 11\right) 4^{\circ} \mathrm{C}$ for $\bullet \min$.

12.138 DCAP RD Mix: $1 \mu \mathrm{l}$ EcoR5, $12 \mu \mathrm{l}$ PCR product; Incubate at $37^{\circ} \mathrm{C}$ for 3 hours

\section{SNP Exploration Thermo-Settings:}

Brassica 27 cycles PCR settings: 1) $94^{\circ} \mathrm{C}$ for $3: 00 \mathrm{~min}$, 2) $94^{\circ} \mathrm{C}$ for $\left.30 \mathrm{~s}, 3\right) 50^{\circ} \mathrm{C}$ for $30 \mathrm{~s}, 4) 72^{\circ} \mathrm{C}$ for $\left.50 \mathrm{~s}, 5\right)$ Repeat $\left.2-48 \mathrm{x}, 6\right) 89^{\circ} \mathrm{C}$ for $\left.30 \mathrm{~s}, 7\right) 50^{\circ} \mathrm{C}$ for $30 \mathrm{~s}, 8$ ) $72^{\circ} \mathrm{C}$ for 50s, 9) Repeat $\left.6-827 \mathrm{x}, 10\right) 72^{\circ} \mathrm{C}$ for $\left.3: 00 \mathrm{~min}, 11\right) 4^{\circ} \mathrm{C}$ for $\bullet$.

Brassica 22 cycles PCR settings: 1) $94^{\circ} \mathrm{C}$ for $\left.3: 00 \mathrm{~min}, 2\right) 94^{\circ} \mathrm{C}$ for $\left.30 \mathrm{~s}, 3\right) 50^{\circ} \mathrm{C}$ for $30 \mathrm{~s}, 4) 72{ }^{\circ} \mathrm{C}$ for $\left.50 \mathrm{~s}, 5\right)$ Repeat $\left.2-48 \mathrm{x}, 6\right) 89^{\circ} \mathrm{C}$ for $\left.30 \mathrm{~s}, 7\right) 50^{\circ} \mathrm{C}$ for $30 \mathrm{~s}, 8$ ) $72^{\circ} \mathrm{C}$ for $\left.50 \mathrm{~s}, 9\right)$ Repeat $\left.6-822 \mathrm{x}, 10\right) 72^{\circ} \mathrm{C}$ for $\left.3: 00 \mathrm{~min}, 11\right) 4^{\circ} \mathrm{C}$ for $\bullet$. 